\title{
Soret and Dufour Effects on Peristaltic Transport of MHD Fluid with Variable Viscosity
}

\author{
F. M. Abbasi ${ }^{1}$, T. Hayat ${ }^{1,2, *}$, A. Alsaedi ${ }^{2}$ and B. Ahmed ${ }^{2}$ \\ ${ }^{1}$ Department of Mathematics, Quaid-I-Azam University 45320, Islamabad 44000, Pakistan \\ ${ }^{2}$ Department of Mathematics, Faculty of Science, King Abdulaziz University, P.O. Box 80257, Jeddah 21589, Saudi Arabia
}

Received: 6 Jun. 2013, Revised: 9 Oct. 2013, Accepted: 10 Oct. 2013

Published online: 1 Jan. 2014

\begin{abstract}
This article looks at the Soret and Dufour effects on the magnetohydrodynamic (MHD) peristaltic flow of variable viscosity fluid in a symmetric channel. Analysis is presented in the presence of Ohmic heating. Results for the stream function, temperature and concentration are constructed. The variations of sundry parameters are analyzed.
\end{abstract}

Keywords: Dufour Effects, Peristaltic Transport, MHD Fluid

PACS:47.50-d, 47.15.G-,47.63.-b

\section{Introduction}

The peristaltic flow problems in channel/tube are widely seen in several processes of engineering and physiology. Such flows appear in urine transport from kidney to bladder, swallowing of food through oesophagus, movement of chyme in gastrointestinal tract, spermatozoa transport in the ducts efferentes of male reproductive tract, ovum movement in the female fallopian tube, vasomotion of small blood vessels, water transport from ground to upper branches of tall trees etc. The industrial applications of peristalsis include blood pumps in heart lung machine, sanitary fluid transport and transport of corrosive fluids. Earliest experimental and theoretical models of peristaltic transport in viscous fluids were presented by Latham [1] and Shapiro et al [2]. Since then a vast amount of information on peristalsis under different aspects has been given by various investigators. Few recent studies on the title can be seen through the refs [3, $4,5,6,7,8,9,10,16,17,18]$.

Most of the published papers regarding peristalsis in channels/tubes have been discussed for constant viscosity fluid. Very little attention is given to the situations which can shed light on the peristalsis of variable viscosity fluid. For instance $[11,12,13,14,15]$. To date no information is available on MHD peristaltic flows with variable viscosity and Soret and Dufour effects. Interaction of peristaltic motion with heat transfer is significant in oxygenation and hemodialysis processes. Simultaneous considerations of heat and mass transfer are available in chemical industry problems for example in reservoir engineering regarding thermal recovery process, catalytic reactors, analysis of hot springs in the sea and medicine diffusion in blood veins. Further simultaneous occurrence of heat and mass transfer affecting each other lead to the Soret and Dufour effects. The magnetohydrodynamic character of fluid has a pivotal role in solidification processes of metal and metal alloys, study of nuclear fuel debris, control of underground spreading of chemical wastes and pollution, design of MHD power generators, blood and blood pump machines, treatment of cancer tumor etc. In view of such discussion, the objective of present article is to analyze the MHD peristaltic transport of variable viscosity fluid in a symmetric channel when Soret and Dufour effects are present. Problem formulation invokes the long wavelength and low Reynolds number assumptions. The series solutions are presented and discussed very carefully.

\section{Mathematical analysis}

Let us investigate the magnetohydrodynamic flow of an incompressible viscous fluid in a channel with width $2 a$. The $\bar{X}-$ axis is chosen along the walls of channel and $\bar{Y}-$ axis is taken normal to the $\bar{X}-$ axis. A constant magnetic

\footnotetext{
*Corresponding author e-mail: pensy_t@yahoo.com
} 
field of strength $\mathbf{B}_{0}$ is exerted in the $\bar{Y}-$ direction. Induced magnetic field is not accounted because of small magnetic Reynolds number. Sinusoidal wave propagation on channel walls with constant wave speed $c$ are represented by

$$
H(\bar{X}, \bar{t})=a+b \cos \left(\frac{2 \pi}{\lambda}(\bar{X}-c \bar{t})\right) .
$$

If $(\bar{U}, \bar{V})$ and $(\bar{u}, \bar{v})$ are the velocity components in the laboratory $(\bar{X}, \bar{Y})$ and wave $(\bar{x}, \bar{y})$ frames respectively, then transformations between laboratory and wave frames are

$\bar{x}=\bar{X}-c \bar{t}, \quad \bar{y}=\bar{Y}, \quad \bar{u}=\bar{U}-c, \quad \bar{v}=\bar{V}, \bar{p}(\bar{x}, \bar{y})=\bar{P}(\bar{X}, \bar{Y}, \bar{t})$.

In above expressions $b$ is the wave amplitude, $\lambda$ is the wavelength, $\bar{t}$ is the time and $\bar{P}$ and $\bar{p}$ are the pressures in laboratory and wave frames respectively. Introducing the variables in the forms

$$
\begin{aligned}
& x=\frac{\bar{x}}{\lambda}, y=\frac{\bar{y}}{a}, u=\frac{\bar{u}}{c}, v=\frac{\bar{v}}{c \delta}, \delta=\frac{a}{\lambda}, h=\frac{H}{a}, d=\frac{b}{a}, p=\frac{a^{2} \bar{p}}{c \lambda \mu_{0}}, \\
& \theta=\frac{T-T_{0}}{T_{0}}, \phi=\frac{C-C_{0}}{C_{0}}, M=\left(\frac{\sigma}{\mu_{0}}\right)^{1 / 2} \mathbf{B}_{0} a, v=\frac{\mu_{0}}{\rho}, \operatorname{Re}=\frac{\rho c a}{\mu_{0}}, \\
& t=\frac{c \bar{t}}{\lambda}, u=\frac{\partial \psi}{\partial y}, v=-\frac{\partial \psi}{\partial x}, B r=\operatorname{Pr} E, D u=\frac{D C_{0} K_{T}}{C_{s} \zeta \mu_{0} T_{0}}, S r=\frac{\rho D K_{T} T_{0}}{\mu_{0} T_{m} C_{0}} \\
& S c=\frac{\mu_{0}}{\rho D}, E=\frac{c^{2}}{\zeta T_{0}}, \operatorname{Pr}=\frac{\mu \zeta}{K}, \text { and } \mu(y)=\frac{\bar{\mu}(\bar{y})}{\mu_{0}} .
\end{aligned}
$$

the conservation laws of mass, linear momentum, energy and concentration after utilizing long wavelength and low Reynolds number assumptions yield

$$
\begin{gathered}
\frac{d p}{d x}=\frac{\partial}{\partial y}\left(\mu(y) \frac{\partial^{2} \psi}{\partial y^{2}}\right)-M^{2}\left(\frac{\partial \psi}{\partial y}+1\right) \\
\frac{\partial^{2}}{\partial y^{2}}\left(\mu(y) \frac{\partial^{2} \psi}{\partial y^{2}}\right)-M^{2} \frac{\partial \psi^{2}}{\partial y^{2}}=0 \\
0=\frac{\partial^{2} \theta}{\partial y^{2}}+B r \mu(y)\left(\frac{\partial^{2} \psi}{\partial y^{2}}\right)^{2}+B r M^{2}\left(\frac{\partial \psi}{\partial y}+1\right)^{2}+\operatorname{Pr} D u \frac{\partial^{2} \phi}{\partial y^{2}} \\
0=\frac{1}{S c} \frac{\partial^{2} \phi}{\partial y^{2}}+\operatorname{Sr} \frac{\partial^{2} \theta}{\partial y^{2}}
\end{gathered}
$$

where $p$ is the pressure, $C$ the concentration field, $T$ the temperature field, $\sigma$ the electric conductivity, $D$ the mass diffusivity, $K_{T}$ the thermal diffusion ratio, $\zeta$ the specific heat, $C_{S}$ the concentration susceptibility, $K$ the thermal conductivity, $\mu(y)$ the variable viscosity, $\mu_{0}$ the absolute viscosity, $T_{m}$ the fluid mean temperature, $v$ the kinematic viscosity, $M$ the Hartman number, $R e$ the Reynolds number, $B r$ the Brinkman number, $D u$ the Dufour parameter, $S r$ the Soret parameter, $S c$ the Schmidt number, $E$ the Eckret number, $\operatorname{Pr}$ the Prandtl number, $\delta$ the wave number, $\psi$ the stream function, $C_{0}$ and $T_{0}$ the concentration and temperature at the boundary, $\theta$ the dimensionless temperature and $\phi$ the concentration. The boundary conditions are

$$
\begin{gathered}
\psi=0, \quad \frac{\partial^{2} \psi}{\partial y^{2}}=0, \frac{\partial \theta}{\partial y}=0, \quad \frac{\partial \phi}{\partial y}=0, \quad \text { at } y=0, \\
\psi=F, \quad \frac{\partial \psi}{\partial y}=-1, \theta=0, \phi=0, \quad \text { at } y=h, \\
h(x)=1+d \cos (2 \pi x), F=\int_{0}^{h} \frac{\partial \psi}{\partial y} d y,
\end{gathered}
$$

where $h(x)$ is the dimensionless wall shape and $F$ is the dimensionless flow rate in the wave frame.

Pressure rise per wavelength $\Delta p_{\lambda}$ is

$$
\Delta p_{\lambda}=\int_{0}^{1} \frac{d p}{d x} d x
$$

The dimensionless expression of space dependent viscosity is [11]

$$
\mu(y)=e^{-\alpha y}=1-\alpha y \quad \alpha \ll 1,
$$

where " $\alpha "$ is the viscosity parameter.

We look for solutions in the series form represented below

$$
\begin{aligned}
\psi & =\psi_{0}+\alpha \psi_{1}+\ldots \\
F & =F_{0}+\alpha F_{1}+\ldots \\
p & =p_{0}+\alpha p_{1}+\ldots
\end{aligned}
$$

Employing the procedure of perturbation method and retaining the results up to order $O(\alpha)$ we have

$$
\begin{gathered}
\psi=A_{1}+\frac{(F+h) \alpha A_{2}}{8(-h M \cosh (h M)+\sinh (h M))^{2}}, \\
\theta=-\frac{B r M^{2} B_{1}}{8(-1+\operatorname{Pr} S c S r D u)(h M \cosh (h M)-\sinh (h M))^{2}} \\
-\frac{B r M \alpha\left[B_{2}+B_{3}+B_{4}-B_{5}+B_{6}-B_{7}+B_{8}\right]}{32(-1+\operatorname{Pr} S c S r D u)(h M \cosh (h M)-\sinh (h M))^{3}}, \\
\phi=-\frac{B r M^{2} S c S r\left[C_{1}-C_{2}+C_{3}\right]}{8(-1+\operatorname{Pr} S c S r D u)(h M \cosh (h M)-\sinh (h M))^{2}} \\
+\frac{B r M S c S r \alpha\left[C_{4}+C_{5}+2\left(C_{6}+C_{7}+\frac{1}{4} M\left(C_{8}+C_{9}-C_{10}\right)-C_{11}-C_{12}+C_{13}\right)\right]}{64(-1+\operatorname{Pr} S c S r D u)(h M \cosh (h M)-\sinh (h M))^{3}},
\end{gathered}
$$

where the involved $\mathrm{A}_{i}(i=1,2), \mathrm{B}_{j}(j=1-8)$ and $\mathrm{C}_{k}(k=1-13)$ are presented in the Appendix. The heat transfer coefficient is a vital quantity to be analyzed in the problems of heat transfer as it incorporates the geometry of the problems in to the analysis of heat transfer. The heat transfer coefficient for this problem is defined as follows:

$$
Z=h_{x} \theta_{y} .
$$




\section{Discussion}

This section describes the impacts of pertinent parameters on the temperature and concentration. Here 3-D graphs are explicitly plotted in order to analyze the quantities of interest in a more detailed manner. These types of graphs allow the readers to analyze the physical quantities continuously within a given domain which is not perhaps visible in the 2-D plots. Also these plots explain the changing behavior of physical quantities in a better way. We recall that theme of this study is to point out the influences of Soret and Dufour. Therefore, the results of various parameters associated with velocity are not included. In order to achieve the desired objective, we present the plots in such a way that the left panels (all the "a" parts of the Figs.) are for constant viscosity (i.e. for $\alpha=0.0$ ) and the right ones (all the "b" parts of the Figs.) are for variable viscosity (i.e. for $\alpha=0.4$ ). It is observed in all the graphs related to temperature that the rise in temperature for variable viscosity fluid is relatively slower when compared with fluid having constant viscosity. Here Figs. 1-5 are drawn for temperature whereas the Figs. 6-10 show variation of concentration. Temperature increases with an increase in M (Fig. 1). Further an increase in temperature is abrupt in view of Ohmic heating. The variations of $\mathrm{Du}, \mathrm{Sr}, \mathrm{Sc}$ and $\mathrm{Br}$ on the temperature are displayed in the Figs. 2-5. These Figs. indicate that there is an increase in temperature by increasing $\mathrm{Du}, \mathrm{Sr}, \mathrm{Sc}$ and $\mathrm{Br}$. It is also found that an increase in temperature is more for $\mathrm{Br}$ when compared to the other parameters.

Figs. 6-10 are presented to examine the behavior of embedded parameters on the concentration. Decrease in concentration is observed when $\mathrm{M}$ and $\mathrm{Du}$ increase (see Figs. 6 \& 7). Concentration also decreases when Sr, Sc and $\mathrm{Br}$ are increased (Figs. 8-10). Again through these Figs. it is clear that decrease in concentration is abrupt for variation in $\mathrm{M}, \mathrm{Sc}$ and $\mathrm{Sr}$ but is slow for the case of $\mathrm{Du}$. Further it is seen that the fluid with variable viscosity has a higher value of concentration compared to that of fluid with constant viscosity.

Behavior of heat transfer coefficient $\mathrm{Z}$ for various parameters is shown in the Figs. 11-15. As expected, Z shows an oscillatory behavior which is because of peristalsis. It is also noted that there is no variation in $\mathrm{Z}$ for amplitude ratio ( $\mathrm{d}$ ) between 0 and 0.3 . It is observed from Fig. 11 that $Z$ increases when $M$ is increased. The absolute values of $\mathrm{Z}$ in variable viscosity fluid are more than the constant viscosity fluid. Figs $12-15$ show that $\mathrm{Z}$ increases with the increase in $\mathrm{Du}, \mathrm{Sr}, \mathrm{Sc}$ and $\mathrm{Br}$. Effects of $\mathrm{Du}, \mathrm{Sr}, \mathrm{Sc}$ and $\mathrm{Br}$ on $\mathrm{Z}$ are opposite to that of $\mathrm{M}$.
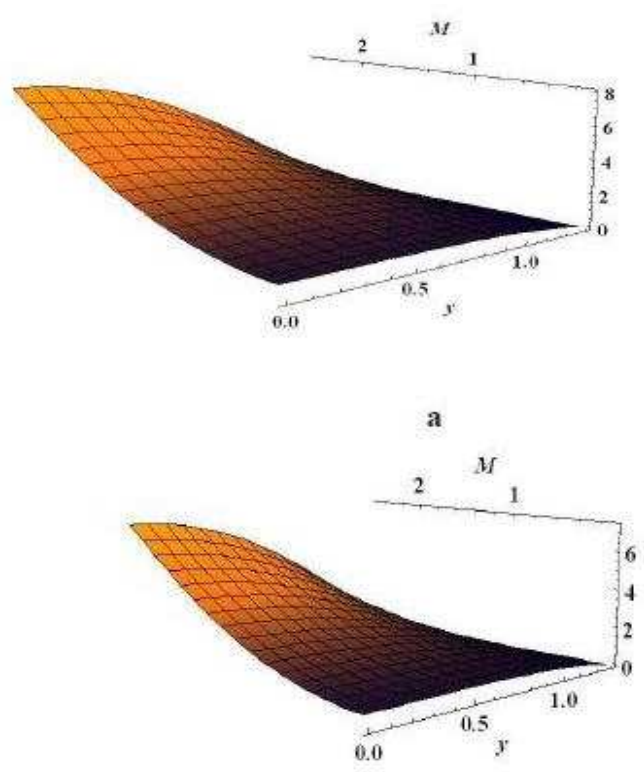

b

Fig. 1: ( $\mathrm{a}$ and b) Effect of $\mathrm{M}$ on $\theta$ when $\mathrm{Du}=0.5, \mathrm{Sr}=0.5, \mathrm{Sc}=0.5$, $d=0.3, \eta=1.4, \mathrm{Br}=0.5$ and $\mathrm{x}=0$.

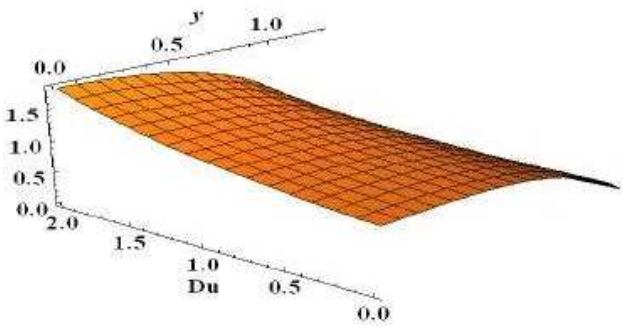

a

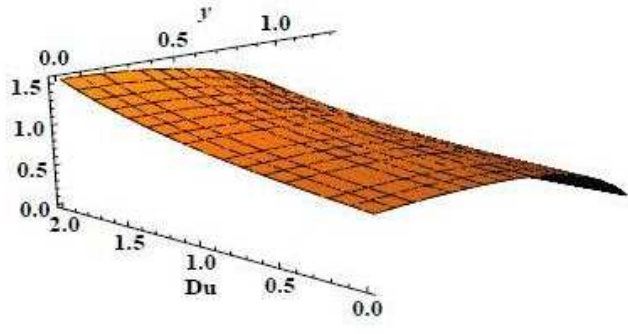

b

Fig. 2: (a and b) Effect of Du on $\theta$ when $\mathrm{M}=0.5, \mathrm{Sr}=0.5, \mathrm{Sc}=0.5$, $d=0.3, \eta=1.4, \mathrm{Br}=0.5$ and $\mathrm{x}=0$. 

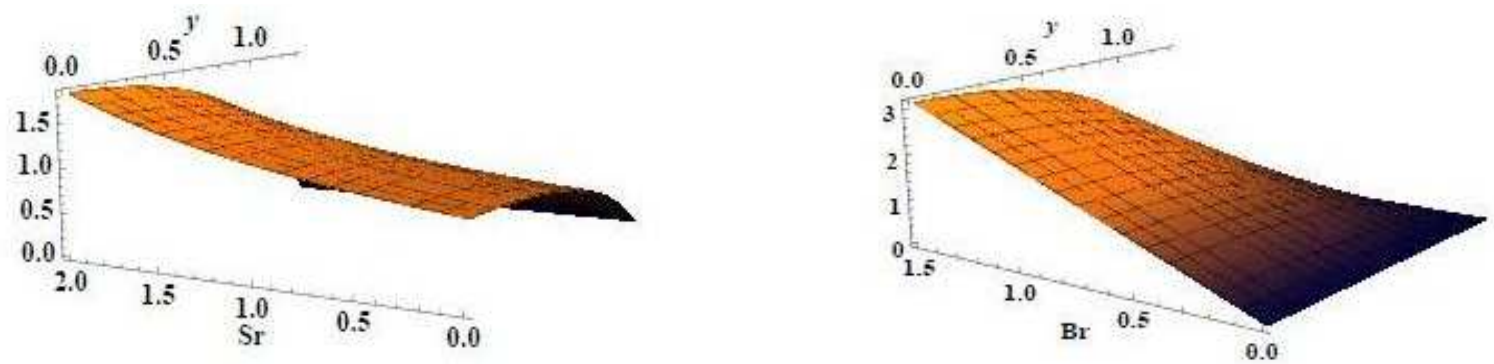

a

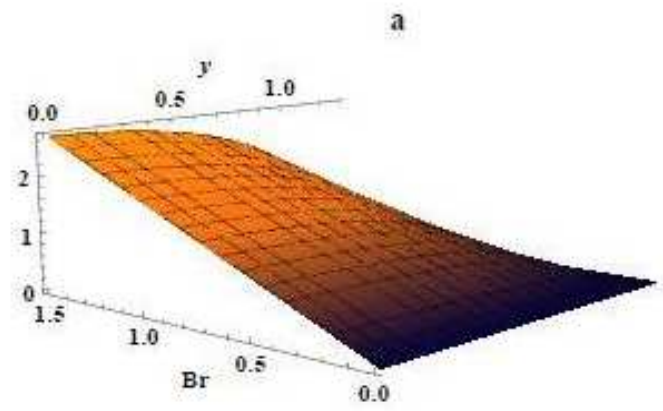

b

b

Fig. 3: ( $\mathrm{a}$ and $\mathrm{b}$ ) Effect of $\mathrm{Sr}$ on $\theta$ when $\mathrm{Du}=0.5, \mathrm{M}=0.5, \mathrm{Sc}=0.5$, $d=0.3, \eta=1.4, \mathrm{Br}=0.5$ and $\mathrm{x}=0$.

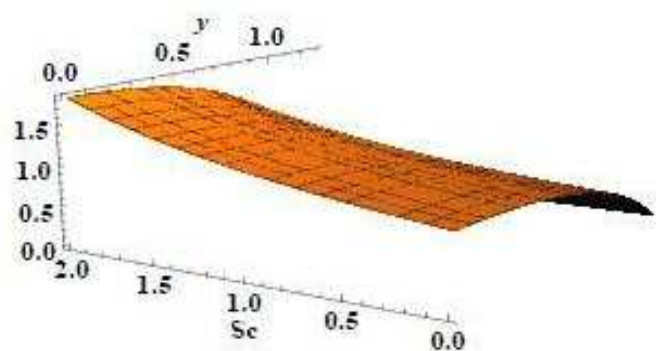

a

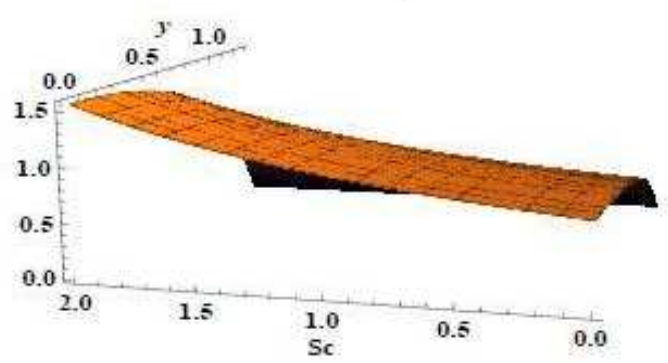

b

Fig. 4: (a and b) Effect of $S c$ on $\theta$ when $D u=0.5, S r=0.5, M=0.5$, $d=0.3, \eta=1.4, \mathrm{Br}=0.5$ and $\mathrm{x}=0$.
Fig. 5: (a and b) Effect of $\mathrm{Br}$ on $\theta$ when $\mathrm{Du}=0.5, \mathrm{Sr}=0.5, \mathrm{M}=0.5$, $d=0.3, \eta=1.4, \mathrm{Sc}=0.5$ and $\mathrm{x}=0$.

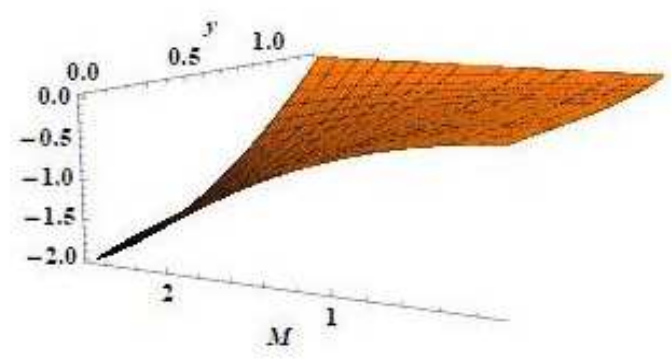

a

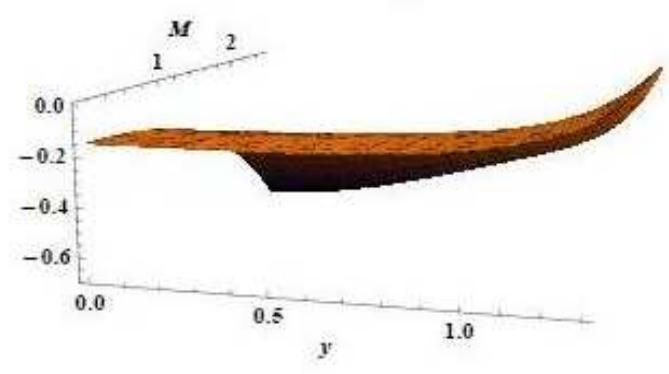

b

Fig. 6: (a and b) Effect of $\mathrm{M}$ on $\phi$ when $\mathrm{Du}=0.5, \mathrm{Sr}=0.5, \mathrm{Sc}=0.5$, $d=0.3, \eta=1.4, \mathrm{Br}=0.5$ and $\mathrm{x}=0$. 


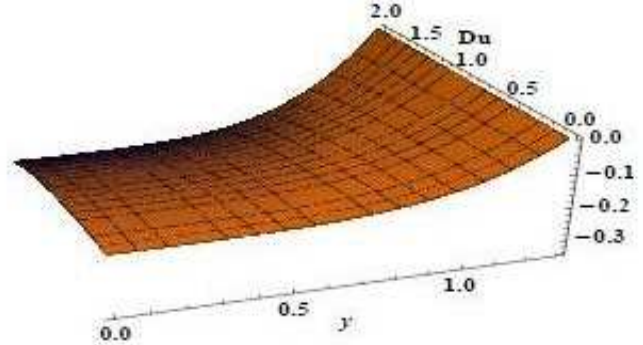

a

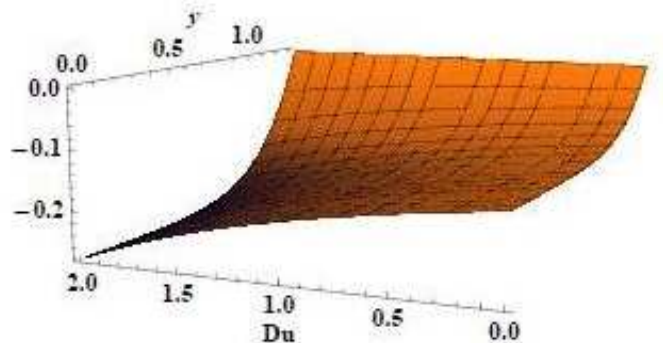

b

Fig. 7: ( $\mathrm{a}$ and $\mathrm{b}$ ) Effect of Du on $\phi$ when $\mathrm{M}=0.5, \mathrm{Sr}=0.5, \mathrm{Sc}=0.5$, $d=0.3, \eta=1.4, \mathrm{Br}=0.5$ and $\mathrm{x}=0$.

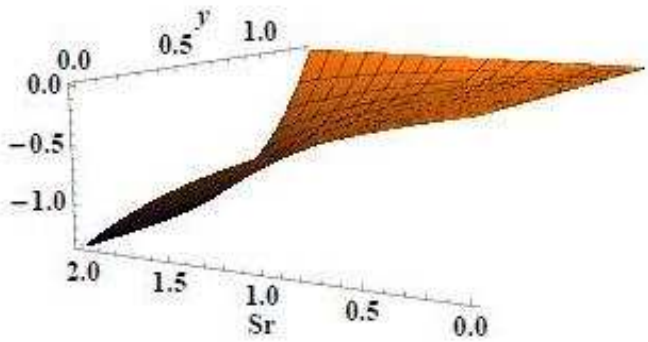

a

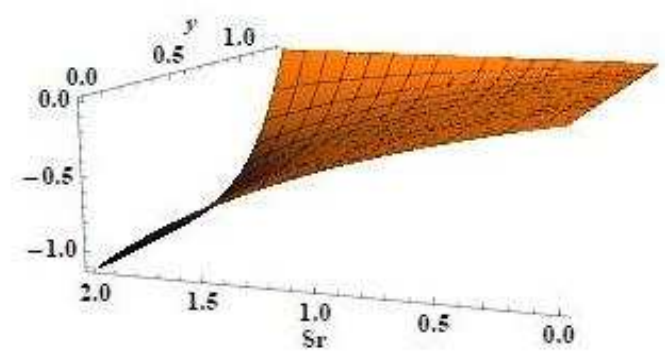

b

Fig. 8: (a and b) Effect of $\mathrm{Sr}$ on $\phi$ when $\mathrm{Du}=0.5, \mathrm{M}=0.5, \mathrm{Sc}=0.5$, $\phi=0.3, \eta=1.4, \mathrm{Br}=0.5$ and $\mathrm{x}=0$.

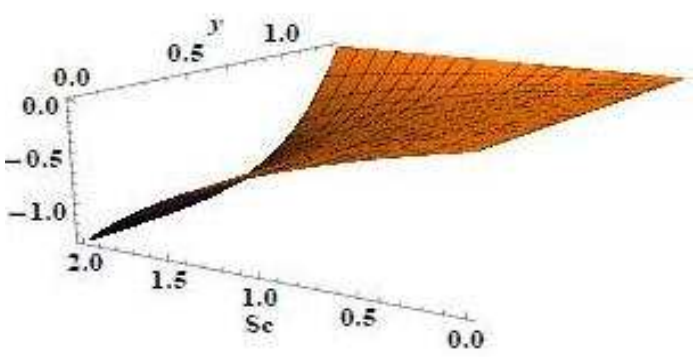

a

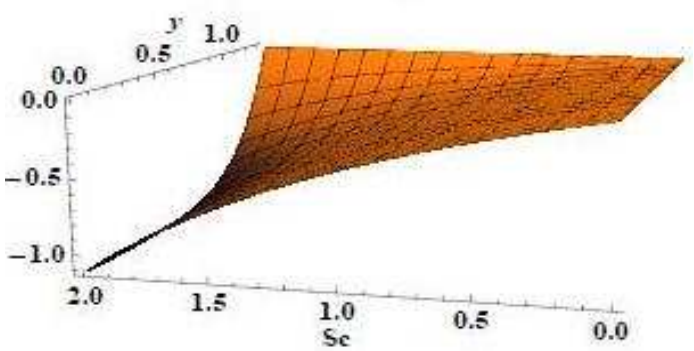

b

Fig. 9: ( $\mathrm{a}$ and b) Effect of $\mathrm{Sc}$ on $\phi$ when $\mathrm{Du}=0.5, \mathrm{Sr}=0.5, \mathrm{M}=0.5$, $d=0.3, \eta=1.4, \mathrm{Br}=0.5$ and $\mathrm{x}=0$.

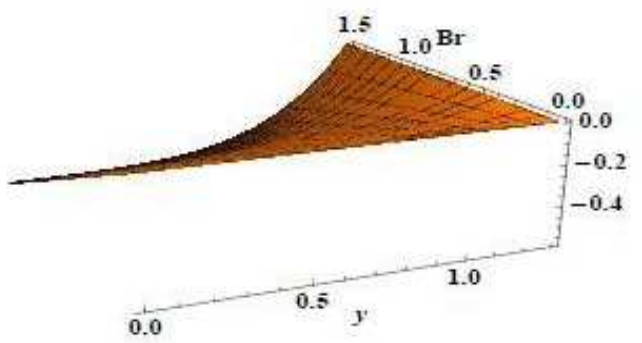

a

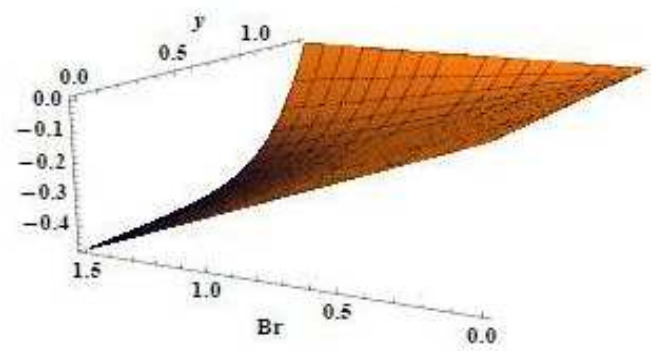

b

Fig. 10: ( $\mathrm{a}$ and $\mathrm{b}$ ) Effect of $\mathrm{Br}$ on $\phi$ when $\mathrm{Du}=0.5, \mathrm{Sr}=0.5, \mathrm{M}=0.5$, $d=0.3, \eta=1.4, \mathrm{Sc}=0.5$ and $\mathrm{x}=0$. 

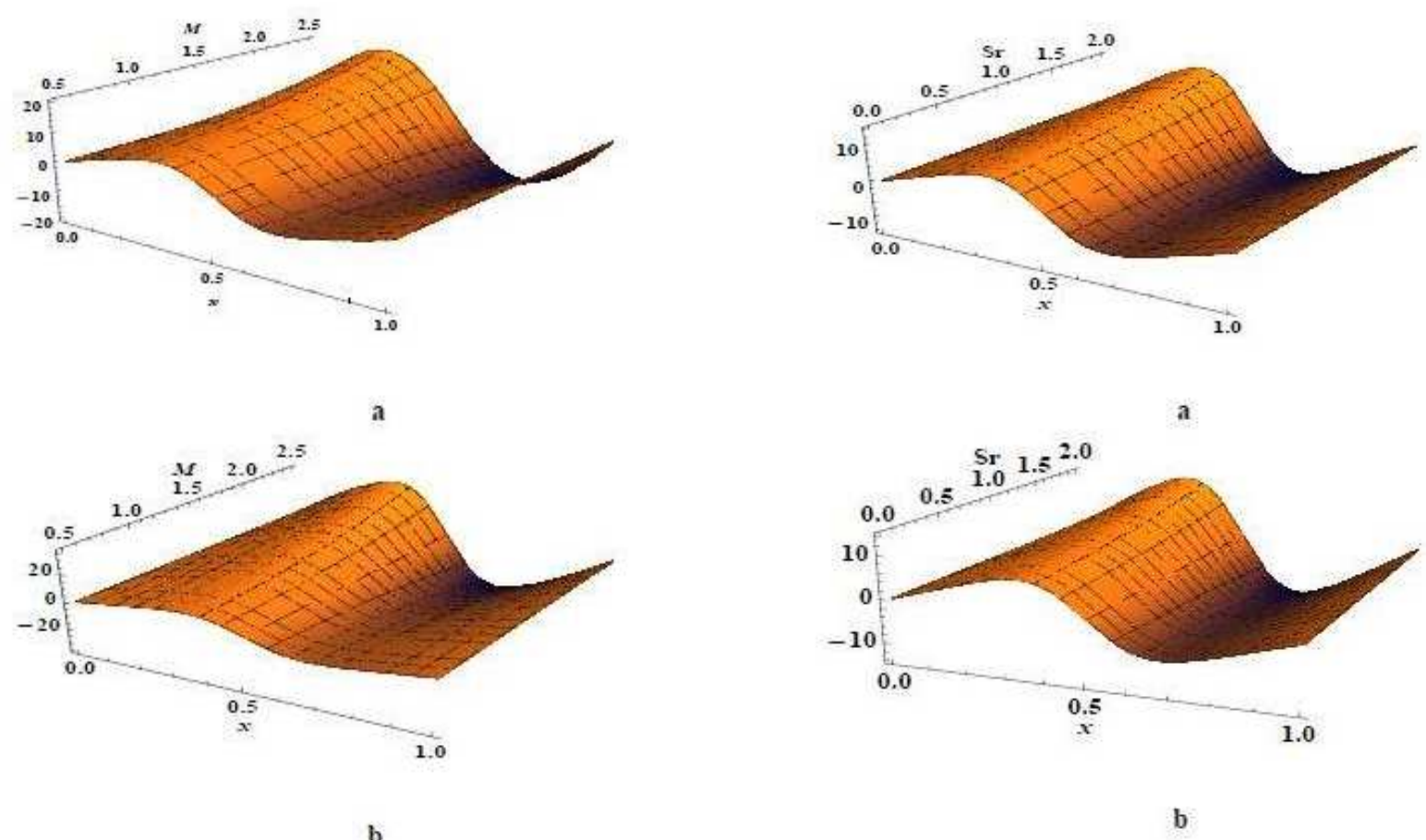

Fig. 11: ( $\mathrm{a}$ and $\mathrm{b}$ ) Effect of $\mathrm{M}$ on $Z$ when $\mathrm{Du}=0.5, \mathrm{Sr}=0.5, \mathrm{M}=0.5$, $d=0.3, \eta=1.4$ and $\mathrm{Br}=0.5$.

Fig. 13: ( $\mathrm{a}$ and b) Effect of $\mathrm{Sr}$ on $Z$ when $\mathrm{Sc}=0.5, \mathrm{Du}=0.5, \mathrm{M}=0.5$, $d=0.3, \eta=1.4$ and $\mathrm{Br}=0.5$.
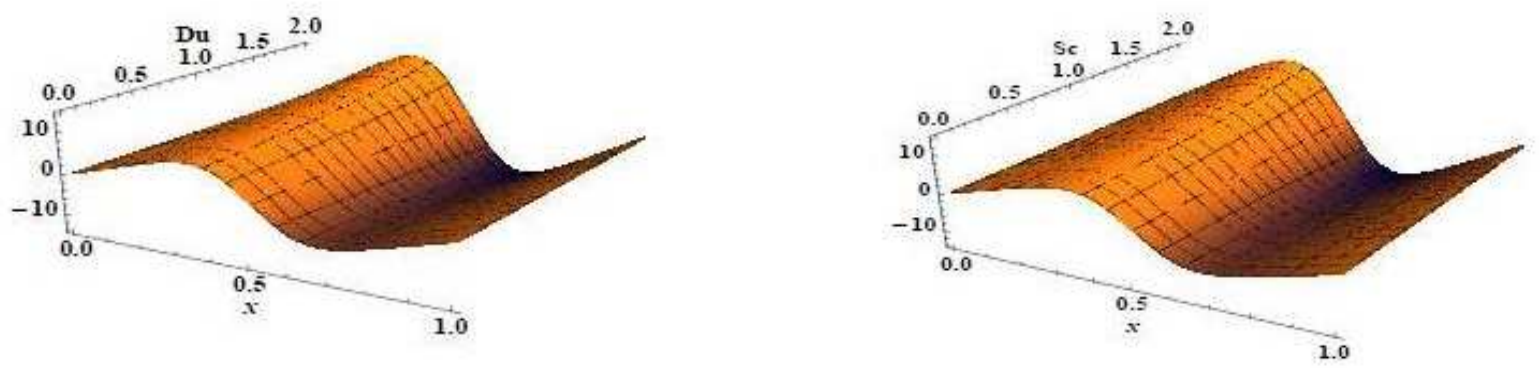

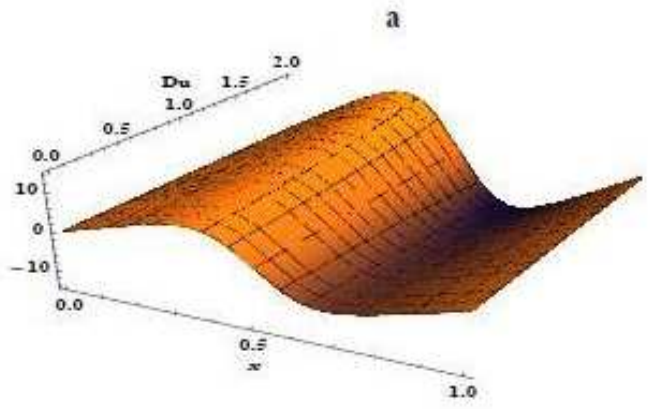

b

Fig. 12: ( $\mathrm{a}$ and b) Effect of $\mathrm{Du}$ on $Z$ when $\mathrm{Sc}=0.5, \mathrm{Sr}=0.5, \mathrm{M}=0.5$, $d=0.3, \eta=1.4$ and $\mathrm{Br}=0.5$.

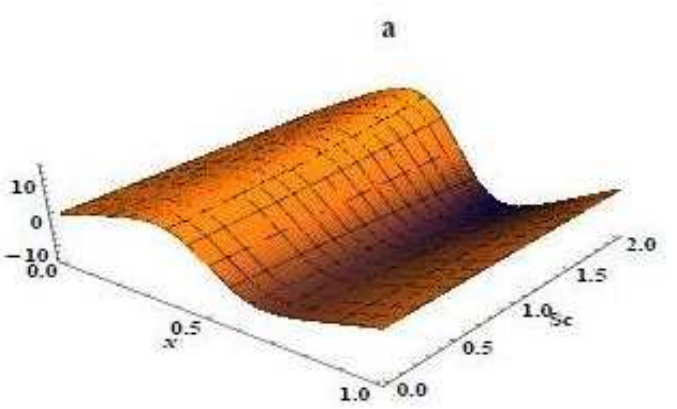

b

Fig. 14: (a and b) Effect of $S c$ on $Z$ when $D u=0.5, S r=0.5, M=0.5$, $d=0.3, \eta=1.4$ and $\mathrm{Br}=0.5$. 


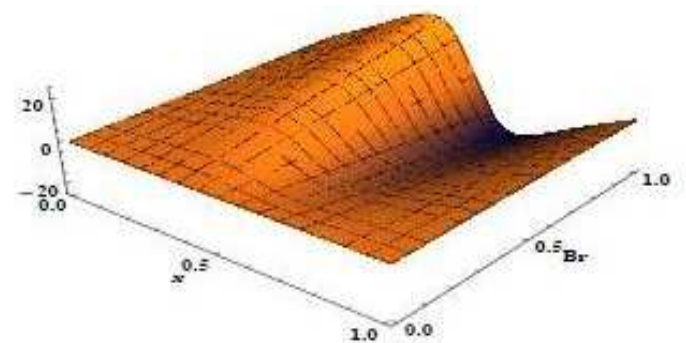

a

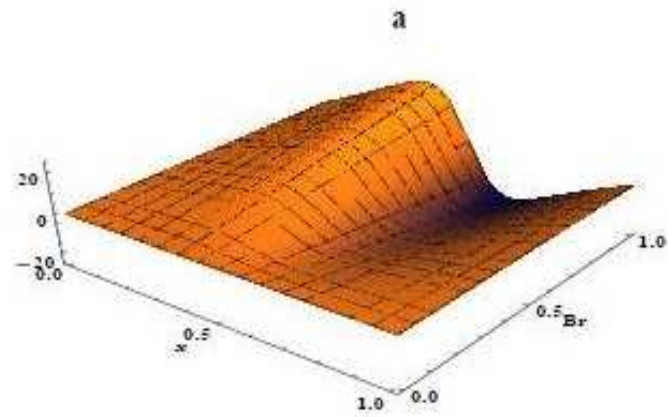

b

Fig. 15: ( $\mathrm{a}$ and b) Effect of $\mathrm{Br}$ on $Z$ when $\mathrm{Sc}=0.5, \mathrm{Sr}=0.5, \mathrm{M}=0.5$, $d=0.3, \eta=1.4$ and $\mathrm{Du}=0.5$.

\section{Acknowledgments:}

We are grateful to the reviewers for the useful suggestions. This paper was funded by the Deanship of Scientific Research (DSR), King Abdulziz University (KAU) under grant no. (9-130/1433 Hi Ci). The authors, therefore, acknowledge technical and financial support of KAU.

\section{References}

[1] T. W. Latham, Fluid motion in a peristaltic pump, M. S. Thesis, Massachusetts Institute of technology, Cambridge, MA, (1966).

[2] A. H. Shapiro, M. Y. Jaffrin and S. L. Weinberg, Peristaltic pumping with long wavelengths and low Reynolds number, J. Fluid Mechanics, 37, 799-825 (1969).

[3] D. Tripathi, S. K. Pandey, S. Das, Peristaltic flow of viscoelastic fluid with fractional Maxwell model through a channel, App. Math. Comp., 215, 3645-3654 (2010).

[4] M. K. Chube, S. K. Pandey and D. Tripathi, Slip effects on peristaltic transport of micropolar fluid, App. Math. Sciences, 4, 2105-2117 (2010).

[5] S. Srinivas, R. Gayathri, M. Kothandapani, Mixed convective heat and mass transfer in an asymmetric channel with peristalsis. Commun. Non-linear Sci. and Numer. Simulat., 16, 1845-1662 (2011).
[6] S. Nadeem, N. S. Akbar, Effects of temperature dependant viscosity on peristaltic flow of a Jeffery-six constsnt fluid in a non-uniform vertical tube, Commun. Non-linear Sci. and Numer. Simulat., 15, 3950-3964 (2010).

[7] Y. Abd Elmaboud, Kh. S. Mekheimer, Non-linear peristaltic transport of a second-order fluid through a porous medium, App. Math. Mode., 35, 2695-2710 (2011).

[8] T. Hayat and S. Hina, The influence of wall properties on the MHD peristaltic flow of a Maxwell fluid with heat and mass transfer, Non-linear analysis: Real world App., 11, 31553169 (2010).

[9] T. Hayat, S. Hina and N. Ali, Simultanious effects of slip and heat transfer on peristaltic flow, Commun. Non-linear Sci. and Numer. Simulat., (2011).

[10] T. Hayat, N. Saleem, S. Asghar, M. S. Althothuali, A. Althomaidan, Influence of induced magnetic field and heat transfer on peristaltic transport of a Carreau fluid, Commun. Non-linear Sci. and Numer. Simulat., 16, 3559-3577 (2011).

[11] N. Ali, Q. Hussain, T. Hayat, S. Asghar, Slip effects on the peristaltic transport of MHD fluid with variable viscosity, Phy. Letters, A 372, 1477-1489 (2008).

[12] D. Tripathi, A mathematical model for the movement of food bolus of varying viscosities through the oesophagus, Acta Astronautica, 69, 429-439 (2011).

[13] T. Hayat and F. M Abbasi, Variable viscosity effects on the peristaltic motion of a third order fluid, Int. J. Numer. Meth. Fluids, 67, 1500-1515 (2011).

[14] Ebaid, A new numerical solution for the MHD peristaltic flow of a bio-fluid with variable viscosity in a circular cylindrical tube via Adomian decomposition method, Phys. Letters, A 372, 5321-5328 (2008)

[15] S. Nadeem and N. S. Akbar, Effects of heat transfer on the peristaltic transport of MHD Newtonian fluid with variable viscosity: Application of Adomian decomposition method, Commun. Non-linear Sci. and Numer. Simulat., 14, 38443855 (2009).

[16] Y. Abd elmaboud and Kh. S. Mekheimer, Unsteady pulsatile flow through a vertical Constricted annulus with heat transfer, Z. Naturforsch. A 67, 185 -194 (2012) .

[17] Kh. S. Mekheimer, S. Z. A. Husseny, Y. Abd Elmaboud, Effect of heat transfer on peristaltic flow in a vertical asymmetric channel: Perturbation solution, Numerical Methods of Partial Differential Equations Journal (NMPDE), 26, 747-770 (2010).

[18] T. Hayat, F. M. Abbasi and S. Obaidat, Peristaltic motion with Soret and Dufour effects, Magnetohydrodynamics, 47, 295-302 (2011) 


\section{Appendix}

$A_{1}=\frac{F M y \cosh (h M)+y \sinh (h M)-(F+h) \sinh (M y)}{h M \cosh (h M)-\sinh (h M)}, A_{2}=y A_{3}+\left(h M y \cosh (h M)+\left(h^{3} M^{2}-y\right) \sinh (h M)\right)$

$2 \sinh (M y), A_{3}=-1+2 h^{2} M^{2}+\cosh (2 h M)+2 M y \cosh (M y)(-h M \cosh (h M)+\sinh (h M))$

$-2 h M \sinh (2 h M), B_{1}=-8 F-2 h(4+h)-2 F+h^{2}(F+2 h) M^{2}-2 h^{2}(F+h)^{2} M^{4}+2$

$\left(1+F(F+2 h) M^{2}+(F+h)^{2} M^{4}\right) y^{2}+$

$\left(F^{2}\left(1+M^{2}\right)+2 F\left(4+h+h M^{2}\right)-2 y^{2}+h\left(8+h\left(3+M^{2}\left(1+2 h^{2}-2 y^{2}\right)\right)\right)\right)$

$\cosh (2 h M)-(F+h)^{2}\left(1+M^{2}\right) \cosh (2 M y)-4 h M\left(2 F+h(2+h)-y^{2}\right) \sinh (2 h M)+$

$16 h(F+h) M \cosh (h M)(M(h-y)+\sinh (M y))-16(F+h) \sinh (h M)(M(h-y)+\sinh (M y))$,

$B_{2}=-8 h^{3}(F+h)(h-y) \sinh (2 h M) M^{4}-\frac{1}{2}(F+h) B_{9} \cosh (h M)-16 h^{4}(F+h) M^{4} \cosh (h M)^{3}$

$+8 h^{3}(F+h) M^{4}(h-y) \cosh (2 h M), B_{3}=\frac{1}{2}(F+h)\left(3(F+h)+3\left(F+h-8 h^{2}\right) M^{2}+8 h^{4} M^{4}\right)$

$\cosh (3 h M)+16 h^{2}(F+h) M^{4} y^{2} \cosh (h M)^{2} \cosh (M y)-2 h(F+h)^{2} M^{2}\left(1+M^{2}\right) y \cosh (h M)$

$\cosh (2 M y), B_{4}=(F+h) M B_{10} \sinh (h M), B_{5}=64 F 1 h^{2} M^{3} \cosh (h M)^{2} \sinh (h M)+2 h^{3}$

$(F+h)^{2} M^{3}\left(1+M^{2}\right) \cosh (2 M y) \sinh (h M)+2(F+h)^{2} M\left(1+M^{2}\right) y \cosh (2 M y) \sinh (h M)+$

$16(F+h) M^{2} y^{2} \cosh (M y) \sinh (h M)^{2}-8 h^{4}(F+h) M^{5}(h-y) \sinh (2 h M), B_{6}=h M B_{11} \cosh (h M)$

$\sinh (2 h M), B_{7}=h M\left(-4 F+F^{2}-4 h+2 F h+h^{2}+(F+h)^{2}\left(1+h^{2}\right) M^{2}+h^{2}(F+h)^{2} M^{4}\right)$

$\sinh (3 h M)-16 h^{2}(F+h) M^{3} y \cosh (h M)^{2} \sinh (M y)+16 h^{3}(F+h) M^{3} \sinh (h M)^{2} \sinh (M y)-$

$16(F+h) M y \sinh (h M)^{2} \sinh (M y)-8 h^{4}(F+h) M^{4} \sinh (2 h M) \sinh (M y), B_{8}=16 h(F+h)$

$M^{2} y \sinh (2 h M) \sinh (M y)+(F+h)^{2}\left(1+M^{2}\right)\left(-3+2 M^{2} y^{2}\right) \sinh (h M) \sinh (2 M y)-h(F+h) M \cosh$

$(h M) B_{12}$,

$B_{9}=F\left(1+M^{2}\right)\left(3+4 h M^{2}\left(-3 h+2 h^{3} M^{2}+2 y-2 M^{2} y^{3}\right)\right)+h$

$\left(3+M^{2}\left(3+4 h\left(h\left(-3+M^{2}\left(-3+2 h\left(1+h+h M^{2}\right)\right)\right)+2(-3+y)+2 M^{2} y-2 M^{2}\left(1+M^{2}\right) y^{3}\right)\right)\right)$,

$B_{10}=-3 h(4+F+h)+h\left(F\left(-3+5 h^{2}\right)+h(-3+h(16+5 h))\right) M^{2}+h^{3}(F+h)\left(5+4 h^{2}\right) M^{4}+$

$4 h^{5}(F+h) M^{6}+4(F+h)\left(1+M^{2}\right) y-4 h^{3}(F+h) M^{4}\left(1+M^{2}\right) y^{2}-4(F+h) M^{2}\left(1+M^{2}\right) y^{3}$,

$B_{11}=-3(F+h)^{2}+\left(-3 F^{2}-6 F h+32 f 1 h+(-3+2 F(8+F)) h^{2}+4(4+F) h^{3}+2 h^{4}\right) M^{2}+2 h^{2}$

$(F+h)^{2} M^{4}, B_{12}=16 M^{2} y^{2} \sinh (M(h-y))+(F+h)\left(1+M^{2}\right)\left(-3+2 M^{2} y^{2}\right) \sinh (2 M y)+16 M^{2} y^{2}$

$\sinh (M(h+y)), C_{1}=2 C_{14}-16 h(F+h) M^{2}(h-y) \cosh (h M)-$

$\left(F^{2}\left(1+M^{2}\right)+2 F\left(4+h+h M^{2}\right)-2 y^{2}+h\left(8+h\left(3+M^{2}\left(1+2 h^{2}-2 y^{2}\right)\right)\right)\right) \cosh (2 h M)$,

$C_{2}=8 F \cosh (M(h-y))-8 h \cosh (M(h-y))+F^{2} \cosh (2 M y)+2 F h \cosh (2 M y)+h^{2} \cosh (2 M y)$

$+F^{2} M^{2} \cosh (2 M y)+2 F h M^{2} \cosh (2 M y)+h^{2} M^{2} \cosh (2 M y)+8(F+h) \cosh (M(h+y))+$

$16 F h M \sinh (h M)+16 h^{2} M \sinh (h M)-16 F M y \sinh (h M)-16 h M y \sinh (h M)$,

$C_{3}=8 F h M \sinh (2 h M)+8 h^{2} M \sinh (2 h M)+4 h^{3} M \sinh (2 h M)-4 h M y^{2} \sinh (2 h M)+$

$8 F h M \sinh (M(h-y))+8 h^{2} M \sinh (M(h-y))-8 h(F+h) M \sinh (M(h+y))$,

$C_{4}=\left(3(F+h)^{2}+\left(3 F^{2}+6 F(1-4 h) h+h(64 f 1+3(1-8 h) h)\right) M^{2}\right) \cosh (3 h M)$,

$C_{5}=2 M \cosh (h M)^{2} C_{15}, C_{6}=-8 h^{3}(F+h) M^{4}(h-y)+2(F+h)^{2} M\left(1+M^{2}\right)\left(h^{3} M^{2}+y\right)$

$\cosh (M y), C_{7}=2 M y \sinh (h M)+16(F+h) M^{2} y^{2} \cosh (M y) \sinh (h M)^{2}, C_{8}=64 F 1 h M$

$\cosh (h M)+32 h^{3}(F+h) M^{3}(h-y) \cosh (2 h M)-64 F 1 h M \cosh (3 h M)$,

$C_{9}=C_{16} \sinh (h M), C_{10}=32 h^{4}(F+h) M^{4}(h-y) \sinh (2 h M)+$

$\left(-h(F+h)\left(-8+5 F+5 h+(5 F+(5-16 h) h) M^{2}\right)+8 F 1\left(4+F+h+\left(F+h-4 h^{2}\right) M^{2}\right)\right)$

$\sinh (3 h M), C_{11}=4 M F 1\left(4-4 h^{2} M^{2}\right)+4 M(F+h)\left(-3 y+h^{2} M^{2}(3 h+y)\right)+4 M$

$\left(F 1\left(4-4 h^{2} M^{2}\right)+4 M(F+h)\left(y+h^{2} M^{2}(-h+y)\right)\right) \cosh (2 h M)+4 M 2 h(F+h) M\left(h^{3} M^{2}-2 y\right)$

$\sinh (2 h M) \sinh (M y)+4 M(F+h)^{2}\left(1+M^{2}\right)\left(-3+2 M^{2} y^{2}\right) \sinh (h M) \sinh (2 M y)$,

$C_{12}=\cosh (h M)(F+h)\left(F\left(1+M^{2}\right)\left(3+4 h M^{2}\left(-3 h+2 h^{3} M^{2}+2 y-2 M^{2} y^{3}\right)\right)\right.$

$+\cosh (h M) h\left(3+M^{2}\left(3+4 h^{2}\left(\begin{array}{c}-3+M^{2}\left(-3+2 h\left(4+h+h M^{2}\right)\right)+2 \cosh (h M)(-3+y) \\ +2 M^{2} y-2 M^{2}\left(1+M^{2}\right) y^{3}\end{array}\right)\right)\right.$,

$C_{13}=64 F 1 h M^{2} \cosh (2 h M)+4 h(F+h)^{2} M^{2}\left(1+M^{2}\right) y \cosh (2 M y)+2 h M$

$\left(-32 F 1 h M^{2} \sinh (2 h M)+(F+h) C_{18}\right), C_{14}=h\left(4+h+h^{3} M^{4}\right)-\left(1+h^{2} M^{4}\right) y^{2}$

$+F^{2} M^{2}\left(1+M^{2}\right)(h-y)(h+y)+2 F\left(2+h M^{2}\left(1+M^{2}\right)(h-y)(h+y)\right), C_{15}=$

$16 h^{2}(F+h) M^{3} y^{2} \cosh (M y)-$

$\left(h(F+h)\left(-8+5 F+5 h+(5 F+(5-16 h) h) M^{2}\right)+8 F 1\left(4+F+h+\left(F+h+4 h^{2}\right) M^{2}\right)\right)$

$\sinh (h M)-8\left(4 f 1\left(-1+h^{2} M^{2}\right)+(F+h)\left(y+h^{2} M^{2}(-h+y)\right)\right) \sinh (M y), C_{16}=8 F 1$

$\left(4+F+h+\left(F+h-4 h^{2}\right) M^{2}\right)+(F+h) C_{17}$,

$C_{17}=-56 h+F h\left(1+M^{2}\right)\left(-13+24 h^{2} M^{2}+16 h^{4} M^{4}\right)+$

$h^{2}\left(-13+M^{2}\left(-13+8 h\left(10+h\left(1+M^{2}\right)\left(3+2 h^{2} M^{2}\right)\right)\right)\right)$

$+16(F+h)\left(1+M^{2}\right) y-16 h^{3}(F+h) M^{4}\left(1+M^{2}\right) y^{2}-16(F+h) M^{2}\left(1+M^{2}\right) y^{3}$,

$C_{18}=16 M^{2} y^{2} \sinh (M(h-y))+(F+h)\left(1+M^{2}\right)\left(-3+2 M^{2} y^{2}\right) \sinh (2 M y)+$

$16 M^{2} y^{2} \sinh (M(h+y))$. 


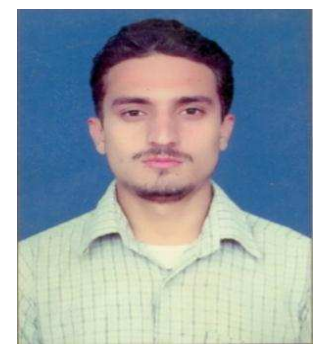
in international Journals of high repute. He is Higher Education Commission (HEC) Pakistans Indigenous scholar since 2011.

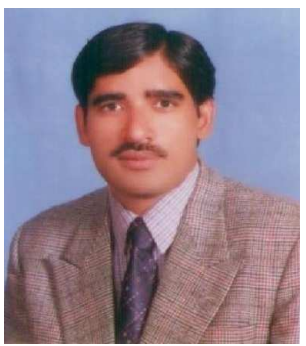

Tasawar Hayat did his Ph.D. in applied mathematics from Quaid-i-Azam University, Islamabad, Pakistan in 1999. Since then, he has been in teaching and research at Quaid-iAzam University, Islamabad, Pakistan. He joined as lecturer in 1998, and has been promoted to Professor.

Besides that, he has been appointed as Distinguished National Professor by the Higher Commission of Pakistan. He has published extensively in Newtonian and non-Newtonian fluid mechanics. He has received a number of national and international awards, and is well known internationally.

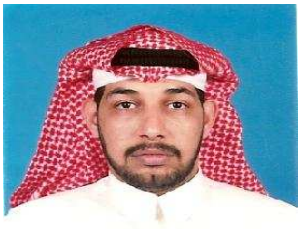

Ahmed Alsaedi obtained his Ph.D. degree from Swansea University, Wales in 2002. He has a broad experience of research in applied mathematics. His fields of interest include dynamical systems, nonlinear analysis involving differential equations, fractional differential equations, boundary value problems, mathematical modeling, biomathematics, Newtonian and Non-Newtonian fluid mechanics. $\mathrm{He}$ has published several articles in peer-reviewed journals. $\mathrm{He}$ has supervised several M.S. students and executed many research projects successfully. He is reviewer of several international journals. He is one of the leading scientists of the King Abdulaziz University (KAU). He served as the chairman of the Mathematics department at KAU and presently he is serving as Director of the Research Program at KAU. Under his great leadership, this program is running quite successfully and it has attracted a large number of ISI Highly Cited Researchers and Distinguished Professors from all over the world. He is also the head of international research group: Nonlinear Analysis and Applied Mathematics (NAAM) at KAU.

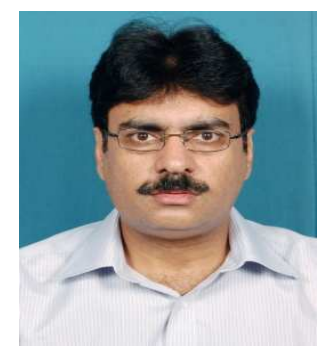

B. Ahmad completed his Ph. D. in 1995 from Quaid-iAzam University, Islamabad, Pakistan. His research interest includes nonlinear ordinary/integro-differential equations, Fractional differential equations, stability analysis and wave motion. He has published extensively. He has been declared as the best researcher of King Abdulaziz University in 2009. At present he has 18 h-index. He is reviewer of several international journals. $\mathrm{He}$ is assistant managing editor of Bulletin Mathematical sciences and associate editor of Advances in Difference Equations. He already completed 11 research projects successfully. He also supervised several research students. 